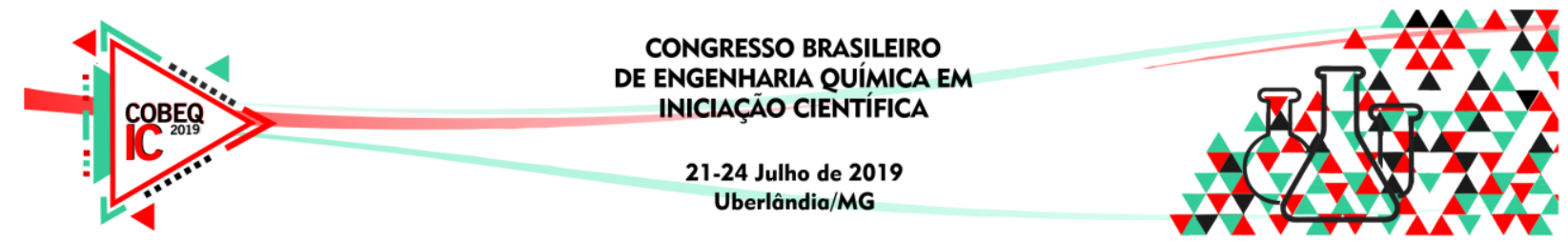

\title{
MICRORREATORES CAPILARES COMO ALTERNATIVA AOS TRADICIONAIS REATORES BATELADA NA SÍNTESE DO INTERMEDIÁRIO FARMACÊUTICO (Z)-5-(4- CLOROBENZILIDENO)TIAZOLIDINA-2,4-DIONA
}

\author{
L. L. RECHE ${ }^{1}$, M. J. AGUIAR ${ }^{2}$ e M. S. A. PALMA ${ }^{2}$ \\ ${ }^{1}$ Faculdades Oswaldo Cruz, Escola Superior de Química, Engenharia Química \\ ${ }^{2}$ Universidade de São Paulo, Faculdade de Ciências Farmacêuticas, Departamento de \\ Tecnologia Bioquímico-Farmacêutica \\ E-mail para contato: msapalma@usp.br
}

\begin{abstract}
RESUMO - Neste trabalho são apresentados os resultados de produção e de número de microrreatores equivalentes, em fluxo contínuo, da reação química entre tiazolidina-2,4-diona (TZD) e 4-clorobenzaldeído, gerando o intermediário farmacêutico (Z)-5-(4-clorobenzilideno)tiazolidina-2,4-diona, que possui atividade farmacológica de combate à diabetes mellitus tipo 2. A partir dos resultados obtidos foi possível concluir que é viável o uso da Tecnologia de Microrreatores (TMR) no lugar dos tradicionais reatores batelada para a realização da síntese proposta.
\end{abstract}

\section{INTRODUÇÃO}

A diabetes mellitus tipo 2 é uma enfermidade caracterizada por hiperglicemia (excesso de glicose no sangue), anormalidades na secreção de insulina, excesso de produção hepática de glicose e resistência à atuação de insulina em músculos esqueléticos, fígado e tecido adiposo (Ortiz e Sansinenea, 2011). No mundo há cerca de 400 milhões de portadores da doença e, segundo estatísticas, em 2040 haverá mais de 640 milhões de enfermos (Peñalver et al., 2016).

O tratamento da diabetes mellitus tipo 2 consiste em uma tríade: adoção de dieta alimentar especial, sob orientação de um nutricionista, prática regular de exercícios físicos, sob tutela de profissional habilitado e administração de medicamentos de atividade antihiperglicemiante, tais como os derivados da tiazolidina-2,4-diona (TZD) (Mishra et al., 2015).

A reação química entre TZD e 4-clorobenzaldeído produz o intermediário farmacêutico (Z)-5-(4-clorobenzilideno)tiazolidina-2,4-diona, conforme mostra o esquema reacional da Figura 1. Essa síntese ocorre pelo mecanismo de condensação de Knoevenagel, através do qual uma carbonila e um metileno ativado interagem quimicamente e formam um aduto com uma ligação $\mathrm{C}=\mathrm{C}$ (Cunha e Santana, 2012). 


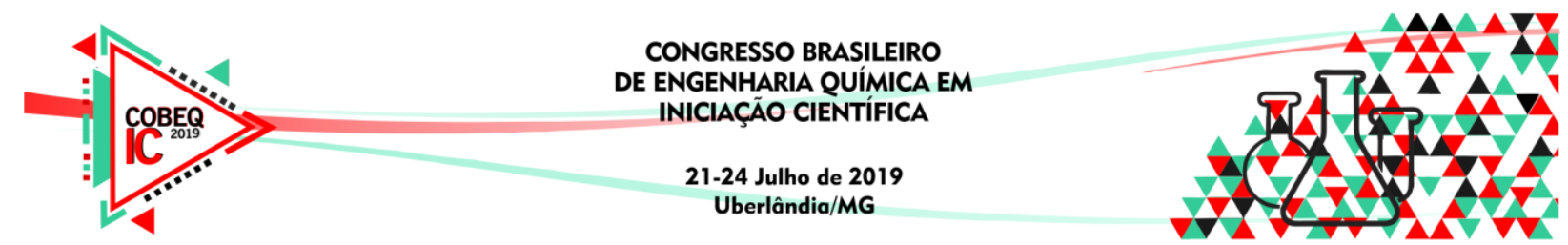

Figura 1 - Esquema da reação entre tiazolidina-2,4-diona (TZD) e 4-clorobenzaldeído formando (Z)-5-(4-clorobenzilideno)tiazolidina-2,4-diona.

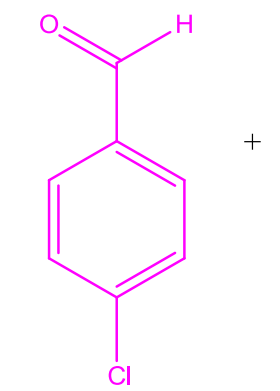

4-clorobenzaldeído

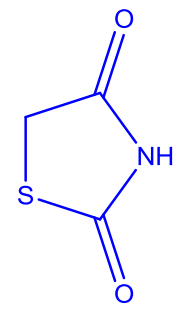

tiazolidina-2,4-diona

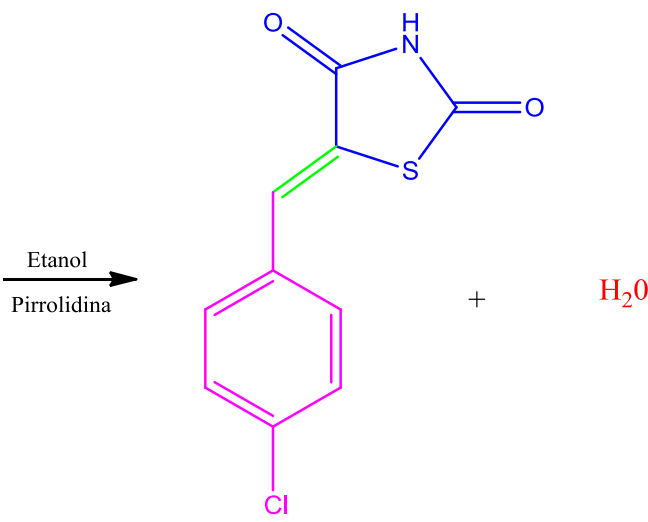

(Z)-5-(4-clorobenzilideno)tiazolidina-2,4-diona

A síntese de produtos farmacêuticos e seus intermediários é tradicionalmente realizada em tanque agitado e processo em batelada. No entanto, a utilização de microrreatores capilares em fluxo traz várias vantagens, principalmente, a redução drástica de tempo para disponibilizar um novo medicamento no mercado (Silva et al., 2019). Os microrreatores capilares são por definição reatores tubulares (PFRs) em tamanho miniatura, com diâmetro médio dos capilares variando entre 10 e $100 \mu \mathrm{m}$ e volume total de $1 \mathrm{~mL}$. Por conta de suas dimensões diminutas o fluxo contínuo ocorre em regime laminar, pois o número de Reynolds (Re) é usualmente baixo, da ordem de 1 a 10 (Watts e Wiles, 2012; Pinheiro et al., 2018). A Figura 2 mostra o microrreator utilizado para a reação química entre TZD e 4clorobenzaldeído.

Figura 2 - Microrreator capilar utilizado na síntese proposta (Asia Syrris ${ }^{\circledR}$ ).

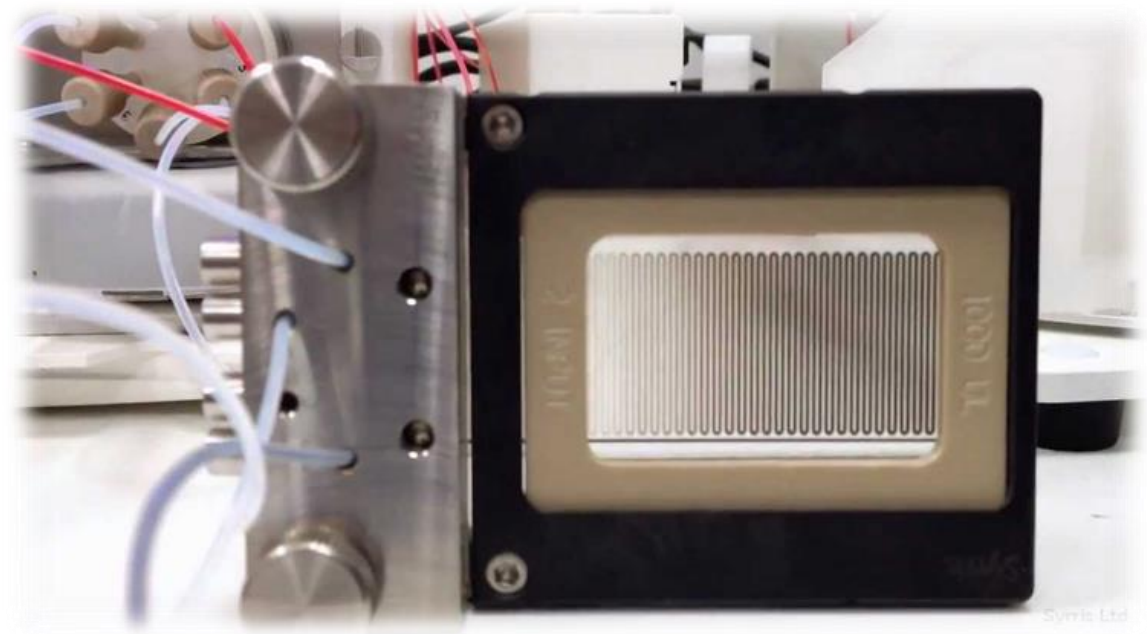

A Tecnologia de Microrreatores (TMR) pode ser utilizada como alternativa aos tradicionais reatores batelada operados na indústria farmacêutica apresentando inúmeras 


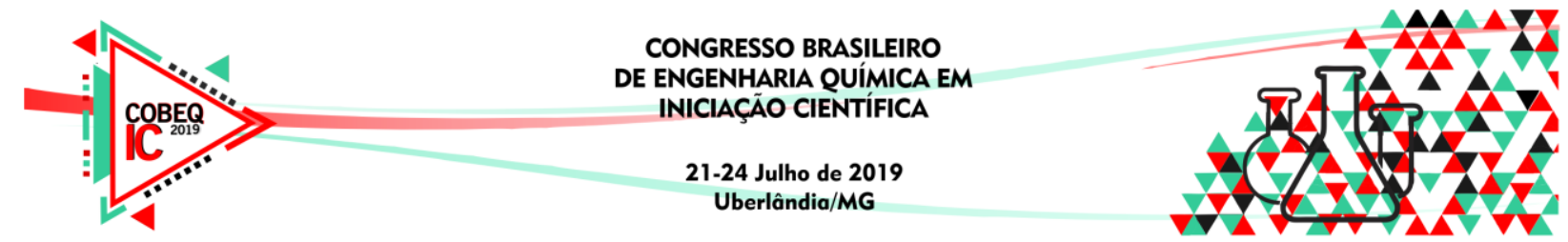

vantagens, tais como: maior eficiência de transferência de calor e de massa, maior difusão dos reagentes, maior seletividade dos produtos, menor geração de resíduos, menor tempo médio de residência (quando comparado ao tempo de reação do reator batelada), maior segurança de processo graças ao controle de pressão, possibilidade de se trabalhar em temperaturas acima do ponto de ebulição normal do solvente e menor espaço físico necessário para instalação e operação (Xu et al., 2015; Jiménez-Gonzalez et al., 2012).

Este trabalho tem como objetivo avaliar a viabilidade do emprego de microrreatores capilares no lugar dos tradicionais reatores batelada na síntese do intermediário farmacêutico (Z)-5-(4-clorobenzilideno)tiazolidina-2,4-diona. Para tal foram avaliados os resultados de produção em microrreator capilar e também o número de microrreatores equivalentes, que indica a quantidade de microrreatores necessários para se atingir a produção máxima alcançada no reator batelada para cada tempo médio de residência estudado.

\section{METODOLOGIA}

O procedimento experimental foi adaptado de Luo et al. (2010). Primeiramente foram preparadas duas soluções, A e B. A solução A consistiu de $4 \mathrm{mmol}(438 \mathrm{mg}, 0,067 \mathrm{M}) \mathrm{de}$ TZD, $30 \mathrm{~mL}$ de etanol $(99,8 \%)$ e $2,4 \mathrm{mmol}(200 \mu \mathrm{L}, 0,040 \mathrm{M})$ de pirrolidina.

A solução B consistiu de $4 \mathrm{mmol}$ (562 mg, 0,067 M) de 4-clorobenzaldeído e $30 \mathrm{~mL}$ de etanol $(99,8 \%)$. Bombas de seringa (Asia Syrris ${ }^{\circledR}$ ) em paralelo foram utilizadas para bombear as duas soluções para o microrreator capilar (Asia Syrris ${ }^{\circledR}$ ) de $1000 \mu \mathrm{L}$.

Em seguida foi ligado o sistema de aquecimento, que consistiu de um banho de óleo de silicone em manta de aquecimento elétrico, e testadas as temperaturas de trabalho $(65,78$, 100, 120, 140 e $160{ }^{\circ} \mathrm{C}$ ). Simultaneamente, o sistema de pressurização foi acionado, garantindo que a pressão de operação ficasse sempre maior que a pressão de vapor do solvente na respectiva temperatura de trabalho, fazendo com que o meio reacional permanecesse no estado líquido.

Foram feitas amostragens de $30 \mu \mathrm{L}$ da corrente de saída do microrreator em vials contendo $1500 \mu \mathrm{L}$ de solução inibidora da reação composta de ACN/ $\mathrm{H}_{2} \mathrm{O} /$ Ácido Acético $(49,70 / 49,70 / 0,60 \%$ v/v) nos tempos médios de residência, $\tau, 2,4,8,12,16$ e 20 min, as quais foram posteriormente analisadas em HPLC-UV (Prominence 20AD da Shimadzu), equipado com coluna Ascentis C18, $5 \mu \mathrm{m}, 25 \mathrm{~cm}$, com métodos analíticos previamente determinados e segundo uma curva de calibração.

A produção no microrreator capilar e o número de microrreatores equivalentes foram calculados pelas Equações 1 e 2, respectivamente:

$$
\begin{gathered}
P M R=Q_{\text {total }} x C_{\text {saída }} \\
N^{\circ} M R=P \text { Batch } / P M R
\end{gathered}
$$





Onde: $P M R=$ Produção no microrreator $(\mathrm{g} / \mathrm{min}) ; Q_{\text {total }}=$ Vazão total do microrreator $(\mathrm{L} / \mathrm{min}) ; C_{\text {saída }}=$ Concentração de produto na saída do microrreator $(\mathrm{g} / \mathrm{L}) ; N^{\circ} M R=$ Número de microrreatores equivalentes; $P$ Batch $=$ Produção no reator batelada $(\mathrm{g} / \mathrm{min})$ para uma batelada de $8 \mathrm{~h}$.

\section{RESULTADOS E DISCUSSÃO}

A Figura 3 apresenta os resultados de produção no microrreator e de número de microrreatores equivalentes em função do tempo médio de residência para as temperaturas de 78 e $140{ }^{\circ} \mathrm{C}$.

Observa-se que o aumento de temperatura acarreta em aumento de produção e diminuição do número de microrreatores equivalentes. Já com o aumento do tempo médio de residência se observa uma situação inversa: diminuição da produção e aumento do número de microrreatores equivalentes.

Figura 3 - Produção no microrreator $(P M R)$ e número de microrreatores equivalentes $\left(N^{\circ} M R\right)$ para a reação entre tiazolidina-2,4-diona e 4-clorobenzaldeído a 78 e $140{ }^{\circ} \mathrm{C}$.

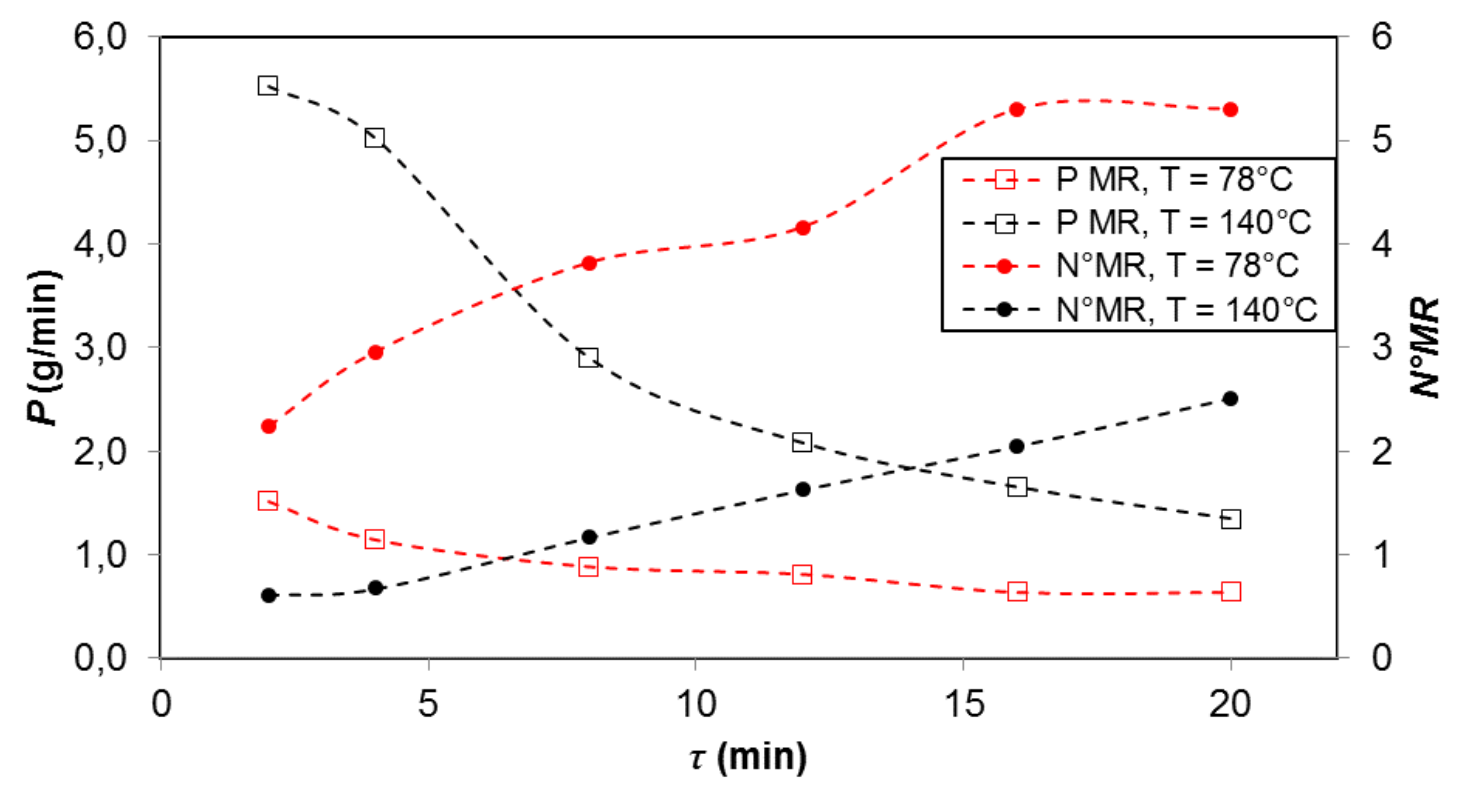

Também foi estimado o número de microrreatores equivalentes para as demais temperaturas estudadas. Os resultados estão compilados na Figura 4 e na Tabela 1.

Nota-se que, no geral, o aumento de temperatura traz correspondente diminuição do número de microrreatores equivalentes, concordando com os resultados apresentados na Figura 3. A concordância também é vista com relação ao aumento do tempo médio de residência, que ocasiona em aumento do número de microrreatores equivalentes. 
Figura 4 - Número de microrreatores equivalentes $\left(N^{\circ} M R\right)$ para todas as temperaturas estudadas.

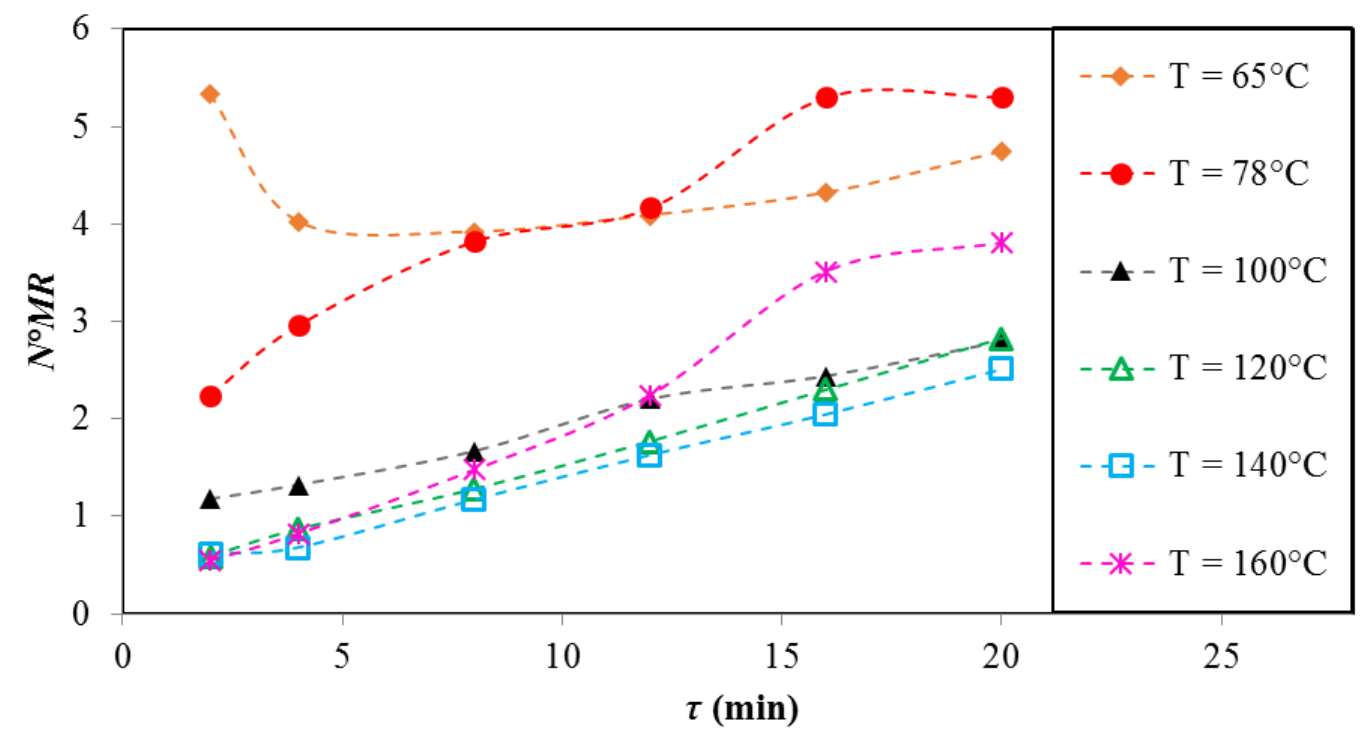

Tabela 1 - Número de microrreatores equivalentes $\left(N^{\circ} M R\right)$ em função da temperatura e do tempo médio de residência $(\tau)$

\begin{tabular}{|c|c|c|c|c|c|c|}
\hline & \multicolumn{7}{|c|}{$\boldsymbol{N}^{\circ} \mathbf{M R}$} \\
\hline & \multicolumn{7}{|c|}{$\boldsymbol{\tau}$ (min) } \\
\hline $\boldsymbol{T}\left({ }^{\circ} \mathbf{C}\right)$ & $\mathbf{2}$ & $\mathbf{4}$ & $\mathbf{8}$ & $\mathbf{1 2}$ & $\mathbf{1 6}$ & $\mathbf{2 0}$ \\
\hline $\mathbf{6 5}$ & 5,33 & 4,02 & 3,92 & 4,09 & 4,32 & 4,74 \\
\hline $\mathbf{7 8}$ & 2,24 & 2,96 & 3,82 & 4,16 & 5,30 & 5,30 \\
\hline $\mathbf{1 0 0}$ & 1,18 & 1,32 & 1,67 & 2,20 & 2,44 & 2,79 \\
\hline $\mathbf{1 2 0}$ & 0,59 & 0,87 & 1,28 & 1,77 & 2,30 & 2,82 \\
\hline $\mathbf{1 4 0}$ & 0,61 & 0,68 & 1,17 & 1,63 & 2,05 & 2,51 \\
\hline $\mathbf{1 6 0}$ & 0,55 & 0,82 & 1,48 & 2,24 & 3,51 & 3,80 \\
\hline
\end{tabular}

\section{CONCLUSÃO}

É possível verificar a influência da temperatura $(T)$ nos resultados obtidos. O aumento de $T$, de 78 para $140{ }^{\circ} \mathrm{C}$ o número de microrreatores equivalentes $\left(N^{\circ} M R\right)$ diminui aproximadamente 2,5 vezes. Tal impacto é percebido também nos valores de produção ( $P$ $M R$ ): para $\tau=2 \mathrm{~min}$, o aumento de $T$ proporciona um aumento de 3,6 vezes de $P M R$, de 1,51 para $5,52 \mathrm{~g} / \mathrm{min}$.

Além disso, os valores de produção, no geral, convergem para o mesmo ponto, de forma que quanto maior o tempo médio de residência mais próximos serão os valores de $P$ $M R$. Esse comportamento do microrreator confirma sua característica de ser indicado para 




reações rápidas, pois quanto menor o tempo médio de residência, maior será a vantagem obtida em termos de $P M R$ e $N^{\circ} M R$. Vale destacar que a condição ótima da reação não será necessariamente aquela com o menor $N^{\circ} M R$, pois a concentração de produto na saída do microrreator $\left(C_{\text {saída }}\right)$ será baixa, mesmo com para produção elevada. Essa situação traria dificuldades para posterior realização de operações unitárias de remoção do intermediário farmacêutico da corrente de saída do microrreator. Estes resultados confirmam a viabilidade de se realizar um processo tradicionalmente feito em reator batelada para um processo em fluxo contínuo em microrreator capilar.

\section{REFERÊNCIAS}

CUNHA, S.; SANTANA, L. L. B. Condensação de Knoevenagel de aldeídos aromáticos com o ácido de meldrum em água: uma aula experimental de química orgânica verde. Quim. Nova, v. 35, p. 642-647, 2012.

JIMÉNEZ-GONZÁleZ, C.; CONSTABLE, D. J. C.; PONDER, C. S. Evaluating the "Greenness" of chemical processes and products in the pharmaceutical industry - a green metrics primer. Chem. Soc. Rev., v. 41, p. 1485-1498, 2012.

LUO, Y.; MA, L.; ZHENG, H.; CHEN, L.; LI, R.; HE, C.; YANG, S.; YE, X.; CHEN, Z.; LI, Z.; GAO, Y.; HAN, J.; HE, G.; YANG, L.; WEI, Y. Discovery of (Z)-5-(4methoxybenzylidene)thiazolidine-2,4-dione, a readily available and orally active glitazone for the treatment of concanavalin A-induced acute liver injury of BALB/c mice. J. Med. Chem., v. 53, n. 1, p. 273-281, 2010.

MISHRA, G.; SACHAN, N.; CHAWLA, P. Synthesis and evaluation of thiazolidinedionecoumarin adducts as antidiabetic, anti-inflammatory and antioxidant agents. Lett. Org. Chem., v. 12, p. 429-445, 2015.

ORTIZ, A.; SANSINENEA, E. Synthetic thiazolidinediones: potential antidiabetic compounds. Curr. Org. Chem., v. 15, p. 108-127, 2011.

PEÑALVER, J. J. M.; TIMÓN, I. M.; COLLANTES, C. S.; GÓMEZ, F. J. C. Update on the treatment of type 2 diabetes mellitus. World J. Diabetes, v. 7, p. 354-395, 2016.

PINHEIRO, D. S.; SILVA, R. R. O.; CALVO, P. V. C.; SILVA, M. F.; CONVERTI, A.; PALMA, M. S. A. Microreactor technology as a tool for the synthesis of a glitazone drug intermediate. Chem. Eng. Technol., v. 41, n. 0, p. 1-9, 2018.

SILVA, R. R. O.; CALVO, P. V. C.; SILVA, M. F.; SOLISIO, C.; CONVERTI, A.; PALMA, M. S. A. Flow synthesis of a thiazolidine drug intermediate in capillary microreactors. Chem. Eng. Technol., v. 42, n. 2, p. 1-10, 2019.

WATTS, P.; WILES, C. Micro reactors, flow reactors and continuous flow synthesis. J. Chem. Res., v. 4, p. 181-193, 2012.

XU, L.; PENG, J.; SRINIVASAKANNANC, C.; CHENA, G.; SHEN, A. Q. Synthesis of copper nanocolloids using a continuous flow based microreactor. Appl. Surf. Sci., v. 355 , p. 1-6, 2015. 